\title{
Serum zonulin as parameter of intestinal permeability in longstanding type 2 diabetes: correlations with metabolism parameter and renal function
}

\begin{abstract}
Background: The raised permeability of the intestinal barrier (IB) is a factor of major pathogenetic relevance for the development of type-1 and type-2 diabetes. Little is known about the extent to which an impaired function of the IB is still detectable even after a relatively long duration of diabetes and which correlations are present between such an impairment and clinical parameters. We investigated these correlations by measuring serum zonulin levels as a parameter of the IB function in people with a longer history of type- 2 diabetes.
\end{abstract}

Methods: In 63 diabetic patients (mean history of diabetes 13 years) and 19 non diabetic controls, the serum zonulin concentrations were measured alongside metabolism parameters (HbAlc, insulin resistance, lipids) and renal function (estimated glomerular filtration rate [eGFR], urinary albumin/creatinine ratio [UACR]). In addition, we also re $\neg$ corded the patients' demographic parameters and drug therapy details.

Results: More than one half of diabetic patients (58\%) exhibited elevated serum zonulin levels compared to nondiabetic controls. Regression analyses showed a significant positive correlation of zonulin concentrations to BMI and $\mathrm{HbA} 1 \mathrm{c}$, but not to insulin resistance or lipids. A significant positive correlation was found between zonulin and eGFR, alongside a significantly negative correlation to proteinuria (UACR). Taking several confounders into consideration, UACR remained an independent factor of influence on the zonulin concentration.

Conclusion: An impaired IB was found in the majority of the diabetic patients even after a long-term duration of the disorder. Whether the detected positive associations between the zonulin concentration and $\mathrm{HbAlc}$ as well as BMI are reasons or consequences of the impaired IB must be investigated further in longitudinal studies. The correlations between circulating zonulin and parameters of kidney function demonstrated here for the first time may indicate that zonulin has effects in other organs too that have to be clarified in further studies.

Keywords: Diabetes mellitus Type 2, intestinal barrier, zonulin, renal function, metabolic endotoxinemea
Volume 5 Issue 2 - 2018

\author{
C Hasslacher, ${ }^{1,2}$ F Kulozik, ${ }^{2}$ I Platten, ${ }^{1,2}$ M \\ Kraft, ${ }^{1,2}$ EG Siegel ${ }^{3}$ \\ 'Clinical Study Department, St. Josefskrankenhaus, Germany \\ ${ }^{2}$ Diabetes Institute, Germany \\ ${ }^{3}$ Department of Internal Medicine, Gastroenterology, \\ Diabetology and Nutrition Medicine, St. Josefskrankenhaus \\ Heidelberg GmbH, Germany
}

Correspondence: Christoph Hasslacher, St. Josefskrankenhaus, Landhausstr. 25, D-69II 5 Heidelberg, Germany, Tel +49 6221 6531970, Fax +4962216531979,

Email c.hasslacher@diabetesinstitut-hd.de

Received: December 14, 2017 | Published: April 02, 2018
Abbreviations: BMI, Body Mass Index; CKD-EPI, Chronic Kidney Disease Epidemiology Collaboration; DPP4 Inhibitors, Dipeptidyl Peptidase 4 inhibitors; eGFR, estimated Glomerular Filtration Rate; HbA1c, Glycated hemoglobin; HOMA-IR, homeostatic model assessment insulin resistance; hsCRP, high sensitive C-Reactive Protein; IB, Intestinal Barrier; IgA, Immunglobulin A; IgG, Immunglobulin G; NYHA, New York Heart Association Classification; UACR, Urinary Albumin/Creatinine Ratio

\section{Introduction}

Besides its involvement in the digestion and resorption of nutrients, one task of the intestine is to act as a barrier regulating the passage of antigens and macromolecules from the environment into the organism. This barrier is to a major extent formed by the intestinal epithelia and its lateral tight junctions which close off the paracellular interstices.
The tight junctions consist of transmembrane barrier proteins such as occludin and claudins, which are bound via proteins of the intracellular cytoskeleton with actin and microtubular structures. ${ }^{1}$ The function of the intestinal barrier (IB) can be modulated by a variety of stimuli. These include nutrients such as gluten, toxins or bacteria or their respective metabolic products absorbed with the nutrition which induce a raised secretion of zonulin through the cells of the lamina propria. ${ }^{1,2}$ Zonulin acts on corresponding receptors of the cytoskeleton of the intestinal cell in such a way that the tight junctions no longer seal off the paracellular interstices, thus enhancing the permeability of the IB. This in turn leads to an increased confrontation of the intestinal immune system or the organism as a whole with potentially pathogenic agents. Not only do disturbances of the IB play a role in the genesis of intestinal disorders, they are also of great importance for the development of systemic diseases, for example of diabetes mellitus. Earlier studies have shown, for example, that the majority 
of people who later suffer from type-1 diabetes previously exhibited raised zonulin concentrations as an indication of an impaired IB. ${ }^{3}$ The disturbed intestinal barrier in association with obesity can lead to low-grade inflammation and subsequently to insulin resistance, thus promoting the development of type- 2 diabetes. ${ }^{4,5}$ To date knowledge about the interrelationship between the function of the IB and clinical factors for patients with a relatively long history of type-2 diabetes is sparse. Therefore, we investigated the serum concentrations of zonulin in this group of patients and to measure their correlation with various clinical parameters.

\section{Materials and methods}

The study was conducted in a population of 63 patients with type-2 diabetes attending the diabetes outpatient clinic of the St. Josefskrankenhaus Heidelberg $\mathrm{GmbH}$ and who fulfilled the following inclusion criteria: Type-2 diabetes with a known history of diabetes of $>2$ years, between 50 and 80 years of age, stable diabetes control, i.e. no change in medication in the previous three months. Exclusion criteria were: Other types of diabetes, acute metabolic imbalance, acute infection, acute or chronic intestinal disorder, antibiotic medication during the previous three months, systemic therapy with cytostatic agents, immunosuppressive drugs, cortisone-containing agents or antirheumatic substances, serious diseases such as tumour diseases, liver cirrhosis, cardiac insufficiency ( $>$ NYHA II), renal insufficiency (eGFR $<30 \mathrm{~mL} / \mathrm{min}$ ), and previous surgery within the gastrointestinal tract. Nineteen nondiabetic and apparently otherwise healthy patients served as controls. The data of all patients were reviewed to record their demographics and their current treatment and complication status. The following laboratory parameters were measured: blood count, $\mathrm{HbA} 1 \mathrm{c}$, glucose, lipids, creatinine, hepatic function parameters, hsCRP, ferritin, insulin, gliadin antibodies ( $\operatorname{IgA}, \operatorname{IgG}$ ), and the urinary albumin/creatinine ratio (UACR). The eGFR parameter was calculated according to the CKD-EPI formula. ${ }^{6}$ All subjects were studied in fasting state. The serum concentration of zonulin was measured using the IKD Zonulin ELISA kit (Immundiagnostik AG, Bensheim, Germany). Measurements were made in duplicate, and the mean values were used for the analysis. As described in the manual of ZONULIN ELISA KIT provided by the manufacturer, mean zonulin concentration in apparent healthy persons (in Germany) is $34 \pm 14 \mathrm{ng} /$ $\mathrm{mL}$. The upper range of zonulin concentration was arbitrarily defined as $>60 \mathrm{ng} / \mathrm{mL}$.

Results were given as mean and standard deviation as well as absolute or relative frequencies, as appropriate. Continuous variables were compared using Student's t-test, Chi square test was used to compare proportions. The relationship between zonulin concentration and clinical parameters was examined by Spearman correlation and linear regression coefficients. Parameters showing significant correlations were then included in a multiple linear regression analysis. Probability values smaller than 0.05 were considered statistically significant. Statistical analyses were performed using the MedCalc software package (version 11.1.1.0, MedCalc Software, Mariakerke, Belgium). The study was approved by the local ethics committee and written informed consent was obtained from all subjects prior to participation.

\section{Results}

The most important demographic findings and laboratory results obtained from the patients and nondiabetic controls in this study are presented in (Table 1). With a mean duration of 13 years, the diabetes patients had already been suffering from the disease for a relatively long period of time. As anticipated, BMI, triglyceride levels, and insulin resistance (HOMA-IR) were higher in these patients as in controls. Due to the higher frequency of statin therapy in the diabetes patients, the cholesterol values were lower in these patients. The remaining laboratory values showed no significant differences. Gliadin antibodies were not detectable in any of the study participants. The exploratory survey of the patients' nutritional habits revealed that none of the study subjects was on a vegetarian or vegan diet. Regarding to the antidiabetic medication: most patients (73\%) were taking metformin, $34.9 \%$ received DPP4 inhibitors or incretin mimetics and $17.5 \%$ sulfonylurea agents. One half of the patients $(55.6 \%)$ were on insulin therapy.

The mean zonulin concentrations in diabetes patients were at $58.8 \pm 15.4 \mathrm{ng} / \mathrm{mL}$ significantly higher than those measured in nondiabetic controls $(42.2 \pm 9.5 \mathrm{ng} / \mathrm{mL}$ ); (Figure 1A). Elevated zonulin concentrations, i.e. $>60 \mathrm{ng} / \mathrm{mL}$, were detected in over one half of the patients (Figure 1B). The results of the regression analyses between zonulin concentrations of diabetic patients and various demographic and laboratory parameters are shown in (Table 2). Zonulin concentrations increased proportionally to increasing obesity and increasing HbAlc levels, but decreased with rising age (Figure 2A). The correlation between zonulin concentration and the logarithmized hsCRP values was only of borderline significance $(\mathrm{p}=0.067)$. No correlation was found between the zonulin concentration and other metabolism parameters such as insulin resistance (HOMA-IR) and the lipid values. A significant correlation was detected between zonulin and renal-function parameters, for example a significant-positive correlation between zonulin concentration and eGFR and a negative correlation between zonulin and UACR (Figure 2B). In multiple regression analyses under the inclusion of age, BMI, HbAlc, eGFR, and UACR as independent variables, only the correlation between zonulin and UACR remained significant (Table 3). No correlation was detected between the zonulin concentration and the type of diabetes therapy. Here the zonulin concentrations in patients with and without insulin $(60.9 \pm 16.3$ vs $62.1 \pm 12.2 \mathrm{ng} / \mathrm{mL})$ and respectively with and without incretin-based therapy ( $63.1 \pm 9.8$ vs $60.6 \pm 16.5 \mathrm{ng} / \mathrm{mL})$ did not differ.

Table I Demographic characteristics and laboratory results obtained in diabetic patients and controls

\begin{tabular}{|c|c|c|c|c|c|c|}
\hline & & \multicolumn{2}{|c|}{ Diabetic Patients } & \multicolumn{2}{|c|}{ Controls } & \multirow[t]{2}{*}{ p-value } \\
\hline & & Mean & SD & Mean & SD & \\
\hline number & $\mathrm{n}$ & 63 & & 19 & & \\
\hline Female/Male & $\mathrm{n}$ & $27 / 36$ & & $9 / 10$ & & \\
\hline Age & years & 67,3 & 6,1 & 64,6 & 9,5 & 0.14 \\
\hline
\end{tabular}


Table Continued...

\begin{tabular}{|c|c|c|c|c|c|c|}
\hline & & \multicolumn{2}{|c|}{ Diabetic Patients } & \multicolumn{2}{|c|}{ Controls } & \multirow[t]{2}{*}{ p-value } \\
\hline & & Mean & SD & Mean & SD & \\
\hline hsCRP & $\mathrm{mg} / \mathrm{l}$ & 3,8 & 3,4 & 2,4 & 2,1 & 0.09 \\
\hline $\mathrm{HbAlc}$ & $\%$ & 7,6 & $\mathrm{I}, 2$ & 5,6 & 0,3 & 0.0001 \\
\hline HOMA-IR & & 7,7 & 6,8 & 2,9 & $\mathrm{I}, 4$ & 0.003 \\
\hline Triglycerides & $\mathrm{mg} / \mathrm{dl}$ & 174,4 & 88,8 & 115,5 & 67,2 & 0.009 \\
\hline Cholesterol & $\mathrm{mg} / \mathrm{dl}$ & 182,5 & 44,5 & 206,3 & 32,4 & 0.03 \\
\hline HDL-Cholesterol & $\mathrm{mg} / \mathrm{dl}$ & 47,9 & 13,1 & 63,2 & 13,2 & 0.0001 \\
\hline LDL-Cholesterol & $\mathrm{mg} / \mathrm{dl}$ & III & 36,6 & 129,3 & 25,9 & 0.04 \\
\hline eGFR & $\begin{array}{l}\mathrm{ml} / \\
\mathrm{min}\end{array}$ & 76,9 & 20 & 82,7 & 13,8 & 0.24 \\
\hline UACR & $\mathrm{mg} / \mathrm{g}$ & 90,4 & 302,2 & 13,7 & 19,4 & 0.27 \\
\hline Ferritin & $\mu g / l$ & 155,3 & 121,5 & 218,9 & $17||$, & 0.07 \\
\hline Hemoglobin & $\mathrm{g} / \mathrm{dl}$ & $14, \mid$ & $\mathrm{I}, 2$ & 14,6 & $\mathrm{I}, 4$ & 0.13 \\
\hline
\end{tabular}

eGFR, estimated glomerular filtration rate UACR, urinary albumin/creatinine ratio

Table 2 Regression analysis between zonulin levels and different variables

\begin{tabular}{llllll}
\hline Zonulin vs & slope & & \multicolumn{2}{l}{$95 \% \mathbf{C l}$} & p-value \\
\hline age & -0.794 & -1.37 & to & -0.218 & $\mathbf{0 . 0 0 8}$ \\
Diabetes duration & 0.119 & -0.495 & to & 0.733 & 0.699 \\
BMI & 0.729 & 0.141 & to & 1.318 & $\mathbf{0 . 0 1 6}$ \\
HbAlc & 3.493 & 0.458 & to & 6.527 & $\mathbf{0 . 0 2 5}$ \\
HOMA-IR & 0.391 & -0.239 & to & 1.022 & 0.219 \\
Triglycerides & 0.03 & -0.011 & to & 0.071 & 0.148 \\
Cholesterol & 0.044 & -0.038 & to & 0.127 & 0.292 \\
HDL-Cholesterol & -0.099 & -0.381 & to & 0.182 & 0.484 \\
LDL-Cholesterol & 0.031 & -0.071 & to & 0.132 & 0.551 \\
hsCRP (log) & 7.079 & -0.502 & to & 14.659 & 0.067 \\
Ferritin & -0.019 & -0.049 & to & 0.011 & 0.217 \\
Hemoglobin & 0.418 & -2.551 & to & 3.387 & 0.779 \\
eGFR & 0.222 & 0.045 & to & 0.399 & $\mathbf{0 . 0 1 5}$ \\
Albumin/Creatinine-Ratio (log) & -6.154 & -12.105 & to & -2.067 & $\mathbf{0 . 0 4 3}$
\end{tabular}

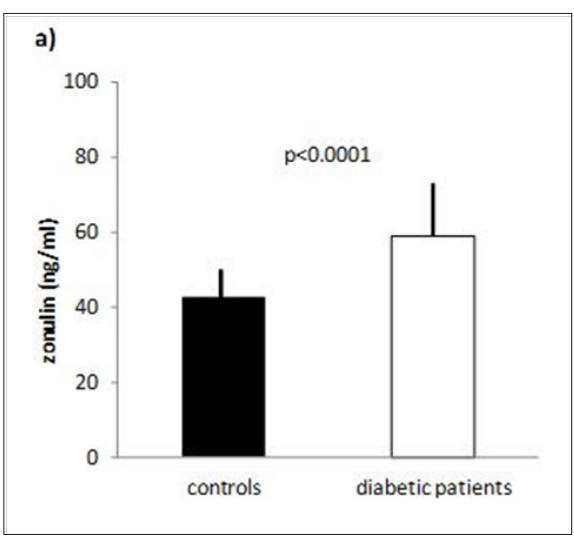

Figure I (A) Mean zonulin concentrations in diabetic patients and nondiabetic controls.

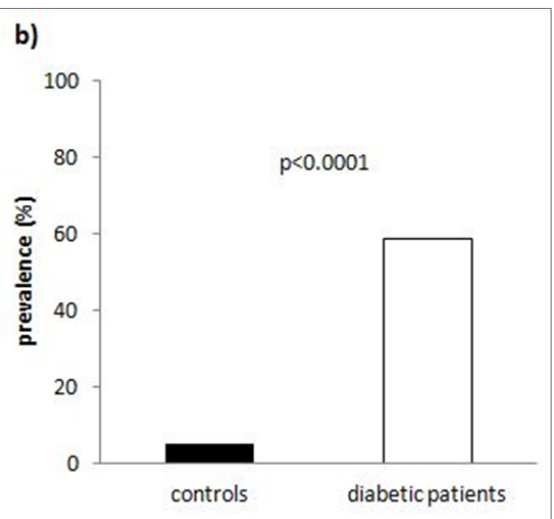

Figure I (B) Prevalence of elevated zonulin concentrations in diabetic patients and nondiabetic controls. 
Table 3 Multiple linear regression analysis with serum zonulin levels as the dependent variable under the inclusion of age, BMI, HbAIc, eGFR, and UACR

as indepen $\neg$ dent variables

\begin{tabular}{lllll}
\hline Independent variables & Coefficient & Std. error & $\mathbf{t}$ & $\mathbf{P}$ \\
\hline age (years) & $-0,1248$ & $0,358 \mathrm{I}$ & $-0,349$ & 0,7286 \\
BMI $\left(\mathrm{kg} / \mathrm{m}^{2}\right)$ & 0,5077 & 0,3326 & $\mathrm{I}, 526$ & 0,1325 \\
HbAlc $(\%)$ & 28,486 & 15,517 & 1,836 & 0,0716 \\
eGFR $\left(\mathrm{ml} / \mathrm{min} / \mathrm{I} .73 \mathrm{~m}^{2}\right)$ & 0,1553 & 0,09977 & $\mathrm{I}, 557$ & $0,125 \mathrm{I}$ \\
log_UACR & $-62,428$ & 28,607 & $-2,182$ & 0,0332 \\
\hline
\end{tabular}
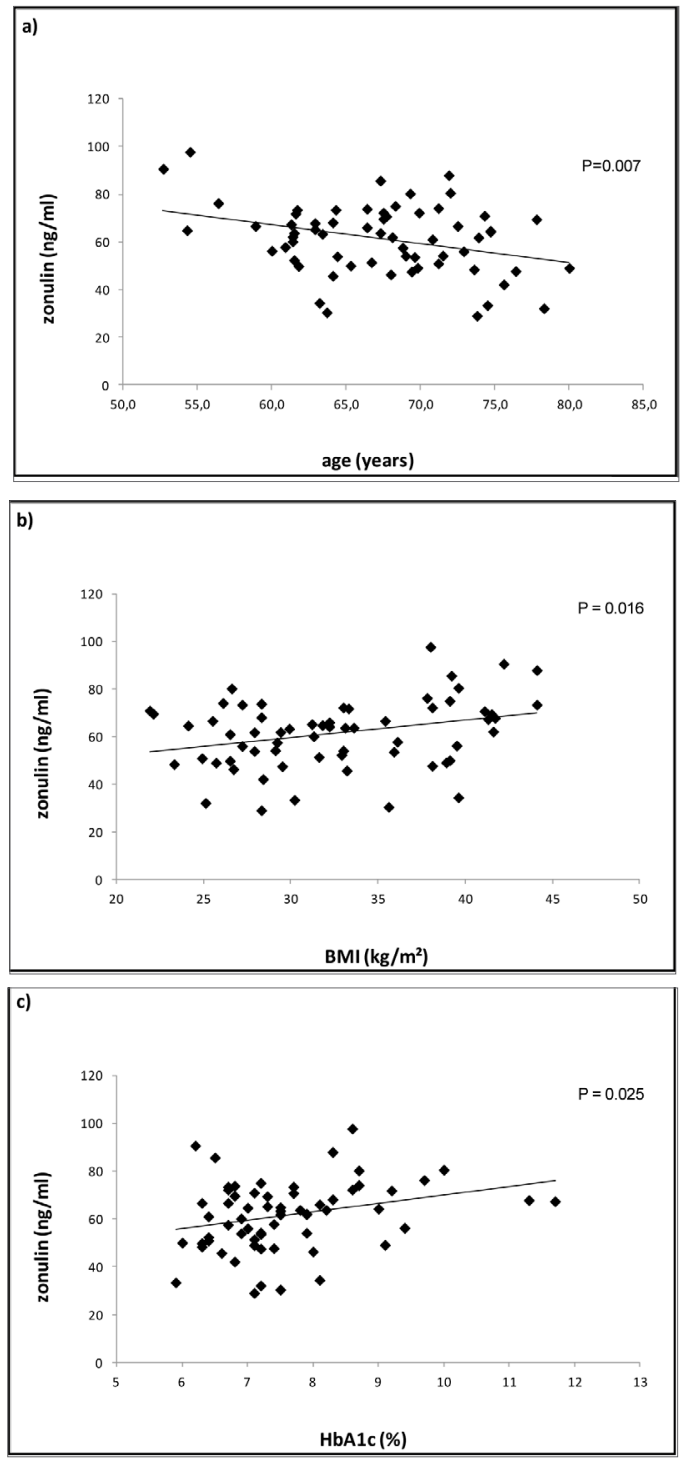

Figure 2 (A) Zonulin concentrations in patients with type-2 diabetes: correlation with age(a), BMI (b), HbAlc (c).
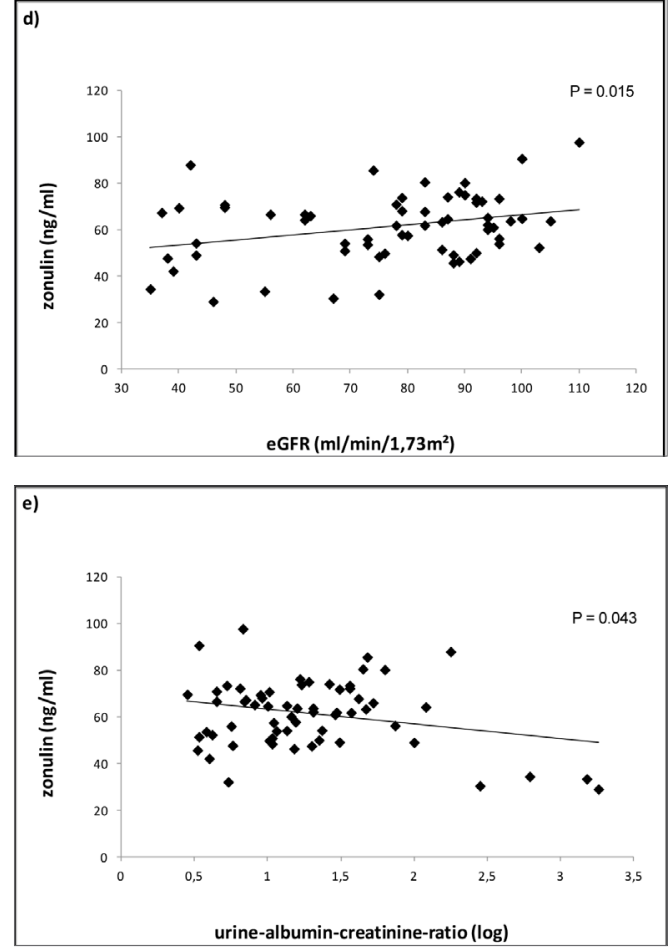

Figure 2 (B) Zonulin concentrations in patients with type-2 diabetes: correlation with eGFR (d) and UACR (e).

\section{Discussion}

Our investigation shows that elevated zonulin concentrations are still detectable as markers of an impaired IB in patients with type-2 diabetes even after a long history of the disorder. The zonulin concentrations correlated positively with $\mathrm{HbA} 1 \mathrm{c}$ and BMI, not, however, with insulin resistance and lipid levels. Described here for the very first time, the significant correlation between zonulin concentration on the one hand and proteinuria and renal function on the other can be interpreted as an indication that zonulin also affects these organ systems.

Zonulin is the only physiological mediator that is known to influence gut barrier permeability by deranging intestinal tight junctions. ${ }^{1,2}$ Serum zonulin levels show a strong correlation with the lactose/mannitol test that is commonly used as a parameter of intestinal barrier function. ${ }^{3,7}$ An impaired IB is associated with the onset of various disorders such as intestinal, liver, or autoimmune diseases as well as obesity and diabetes. ${ }^{1,4,5}$ Regarding type- 1 diabetes, Sapone et al. ${ }^{3}$ found elevated zonulin levels in about $70 \%$ of type- 1 diabetic children that precede the onset of diabetes by about 3-4 years. Regarding the development of type-2 diabetes, Moreno-Navarrete et al. showed that Zonulin concentration associates with obesity and insulin resistance. ${ }^{8}$ Furthermore it has been described that patients with newly diagnosed type-2 diabetes exhibit higher zonulin levels than do patients with impaired or normal glucose tolerance. ${ }^{9,10}$ It has been assumed that impairment of the IB due to overweight or obesity results in an increase in plasma lipopolysaccharides (LPS), i.e. metabolic endotoxemia, that induce the production of proinflammatory 
cytokines and insulin resistance and ultimately lead to diabetes. ${ }^{8,11}$

In our study we investigated type-2 diabetics who had already been suffering from the disorder for a relatively long time. In comparison with non-diabetic controls of similar age, the zonulin concentrations were significantly higher in over one half of the type- 2 diabetic patients $(58 \%)$. Seen from this aspect, not only does an impaired IB appear to be of relevance for the development of type- 2 diabetes, it is also detectable in the further course of the disorder associated with the known risk of the passage of toxins (metabolic endotoxinemia) that implicates a risk of development of cardiovascular complications. ${ }^{12,13}$ We found significantly positive correlations between the zonulin level and influenceable parameters such as $\mathrm{HbA1c}$ and BMI, not, however, with lipid values and insulin resistance or chronic inflammation (hsCRP). To a certain extent, these results contradict those made by other workgroups. ${ }^{9,10}$ But these authors investigated patients with newly manifest diabetes or ones with a shorter history of the disease and coming from other cultural spheres (China, India), meaning that e.g. different nutritional habits also play a role. This may also be a reason for the much lower zonulin concentrations found in these studies compared with our results. Our findings indicate that the correlation between impaired IB and insulin resistance has a lower impact in patients with a relatively long history of diabetes. The extent to which an impaired IB can be influenced by improving the metabolic status or the BMI must be clarified in prospective studies. In the multiple regression analysis involving further confounding factors, these correlations were no longer significant.

Furthermore we detected a significantly positive correlation between renal function, measured as eGFR, and the zonulin concentration and were thus able to confirm the finding recently reported by Dschietzig et al. ${ }^{14}$ In this study, which was conducted in a special group of patients with an automatic implantable cardioverter/ defibrillator, the authors had described such a correlation for the first time, deducing that the low zonulin concentration associated with impaired renal function results from the depletion of zonulin with the urine. We were indeed able to determine a significantly negative correlation between the serum zonulin concentration and the extent of proteinuria measured as UACR in the urine. After considering several other confounders in the investigation, UACR proved to be the sole independent factor influencing the zonulin concentration in the serum. Further investigations must now be conducted to show whether zonulin actually is excreted simply "passively" with the urine, or else whether this protein possesses not only intestinal, but also renal effects.

Limitations of our study include primarily the relatively low number of patients involved and also its observational design. In addition, it was not possible to record detailed findings on nutritional habits, in particular with regard to the uptake of gliadin-containing food, the consumption of alcohol, and the use of pre- or probiotics. Measurements of the serum LPS concentration to determine the risk of toxic organ damage and analyses of the composition of intestinal microbiom to investigate the influence of bacteria on the IB in diabetes would also have been helpful, and such factors should be considered in future studies.

\section{Conclusion}

Our results show that elevated zonulin concentrations in the serum as an expression of an impaired intestinal barrier are a factor of relevance not only for the emergence of type- 2 diabetes, but also remain detectable even after a long history of the disorder and correlate with the quality of the metabolic control and BMI. The correlations reported here between circulating zonulin and renal function/ proteinuria indicates that this protein may possess further effects in other organs that extend beyond the already known influence on the intestinal barrier. This aspect, too, should be clarified in more extensively designed studies.

\section{Acknowledgement}

The study was kindly supported by an unrestricted grant of the Dietmar Hopp Stiftung, St. Leon Rot, Germany

\section{Conflict of interest}

The authors declare no potential conflicts of interest with respect to the research, authorship, and/or publication of the study.

\section{References}

1. Fasano A. Zonulin and its regulation of intestinal barrier function: the biological door to inflammation, autoimmunity, and cancer. Physiol Rev. 2011;91(1):151-175.

2. Wang W, Uzzau S, Goldblum SE, et al. Human zonulin, a potential modulator of intestinal tight junctions. $J$ Cell Sci. 2000;113(24):4435-4440.

3. Sapone A, de Magistris L, Pietzak M, et al. Zonulin upregulation is associated with increased gut permeability in subjects with type 1 diabetes and their relatives. Diabetes. 2006;55(5):1443-1449.

4. de Kort S, Keszthelyi D, Masclee A. Leaky gut and diabetes mellitus: what is the link? Obes Rev. 2011;12:449-458.

5. Fasano A. Intestinal permeability and its regulation by zonulin: diagnostic and therapeutic implications. Clin Gastroenterol Hepatol. 2012; 10(10):1096-1100.

6. Levey AS, Stevens LA, Schmid CH, et al. A new equation to estimate glomerular filtration rate. Intern Med. 2009;150(9):604-612.

7. Marsilio R, D’Antiga L, Zancan L, et al. Simultaneous HPLC determination with light-scattering detection of lactulose and mannitol in studies of intestinal permeability in pediatrics. Clin Chem. 1998;44:1685-1691.

8. Moreno-Navarrete JM, Sabater M, Ortega F, et al. Circulating Zonulin, a marker of intestinal permeability, is increased in association with obesity associated insulin resistance. PloS One. 2012;7(5);e37160

9. Zhang D, Zhang L, Zheng Y, et al. Circulating zonulin levels in newly diagnosed Chinese type 2 diabetes patients. Diabetes Res Clin Pract. 2014;106(2):312-318.

10. Jayashree B, Bibin YS, Prabhu D, et al. Increased circulatory levels of lipopolysaccharide (LPS) and zonulin signify novel biomarkers of proinflammation in patients with type 2 diabetes. Mol Cell Biochem. 2014;388(1-2):203-210.

11. Gomes JMG, Costa J de A, Alfenas R de CG. Metabolic endotoxemia and diabetes mellitus: A systematic review. Metabolism. 2017;68:133-144.

12. Karlsson FH, Fåk F, Nookaew I, et al. Symptomatic atherosclerosis is associated with an altered gut metagenome. Nat Commun. 2012;3:1245.

13. Tang WH, Kitai T, Hazen SL. Gut Microbiota in Cardiovascular Health and Disease. Circulation Res. 2017;120(7):1183-1196.

14. Dschietzig TB, Boschann F, Ruppert J, et al. Plasma Zonulin and its Association with Kidney Function, Severity of Heart Failure, and Metabolic Inflammation. Clin Lab. 2016;62(12):2443-2447. 